\title{
LA ACTIVIDAD TEATRAL EN LOGROÑO ENTRE 1901 Y 1950
}

\author{
M. a Ángel SOMALO FERNÁNDEZ \\ a-somalo@terra.es
}

Resumen: Reconstrucción de la cartelera teatral de Logroño entre 1901 y 1950, atendiendo a las compañías profesionales, con referencias a obras y autores representados, así como a la recepción crítica.

Résumé: Reconstruction de l'activité théâtrale en la ville de Logroño entre 1901 et 1950. Dramaturges et pièces, mise en scène, compagnies de théâtre et valorisation de la critique.

Palabras clave: Cartelera teatral. Historia del teatro representado. Logroño.

Mots clé: Rubrique des affiches. Histoire de la mise en scène. Logroño.

La actividad teatral en Logroño durante los cincuenta primeros años del pasado siglo fue bastante intensa, tanto en el terreno profesional como en el aficionado, a tenor de los datos obtenidos en nuestro estudio. Hay que tener en cuenta que el teatro era visto más como una actividad social, como lugar 
donde ver y ser visto, que como un hecho estrictamente cultural, y que el cine aún no se había convertido, en los primeros años de este periodo, en el competidor en que luego se convirtió, especialmente entre las clases más humildes, por su precio más económico.

Pero aquí vamos a ceñirnos a algunos comentarios sobre las compañías profesionales que presentaron su trabajo en los locales logroñeses durante los años citados.

Los elencos, numerosísimos, que pasaron por la capital riojana son básicamente los mismos que actúan en el resto de ciudades españolas, pues Logroño se encontraba también en el itinerario de las giras que las compañías realizaban durante una época del año. Ante la imposibilidad de citar todas las compañías de calidad demostrada o de gran popularidad en la época, hemos centrado nuestra atención en las diez que visitaron más veces nuestra ciudad; se quedarán en el tintero grupos como los de María Guerrero, María Palou o Catalina Bárcena entre los primeros, y los de Loreto-Chicote o Paco Martínez Soria entre los segundos.

Hay que hacer notar que la cantidad de visitas realizadas no tienen nada que ver con la duración de las estancias; hay compañías, como la de Salvador Orozco, por ejemplo, que están durante mucho más tiempo actuando en los escenarios logroñeses, aunque vinieron menos veces. Esta situación se produce sobre todo en los primeros años, en que las estancias en un teatro llegan a durar incluso meses.

Otro hecho curioso es que, frente a la preferencia del público logroñés por los géneros musicales, ninguna de las compañías que reseñamos cultiva este tipo de teatro; todas ellas ofrecen un repertorio de comedias o dramas, y sólo tiene un carácter excepcional la de Rambal, que ofrece al público una serie de obras de carácter más bien melodramático, acompañadas de un aparato escénico de gran impacto e ilustraciones musicales. Veamos cuáles son estas compañías, sus componentes y las obras que presentaron en los escenarios de Logroño.

\section{COMPAÑÍA DE ANA ADAMUZ}

1.1. La primera vez que esta actriz actuó en Logroño durante el período estudiado lo hace en el teatro Bretón, en el que estuvo desde el 17 al 20 de noviembre de 1922; la denominación de la compañía era: Compañía de comedias Adamuz-González. 
a) Elenco:

Actores: Manuel González (1. ${ }^{\text {er }}$ actor), Adolfo Benedicto.

Actrices: Ana Adamuz (1. actriz), Cerdá.

b) Repertorio:

El caudal de los hijos; Don Juan, buen persona; Que no lo sepa Fernanda; El último pecado.

1.2. La segunda estancia de esta actriz tuvo lugar en el teatro anterior entre el 15 y el 22 de noviembre de 1926, bajo la denominación de Gran compañía cómico-dramática Adamuz-González.

a) Elenco:

Actores: Abolafia, Adolfo Benedicto, Escobar, Manuel González (1. ${ }^{\text {er }}$ actor), Casimiro Hurtado, Manuel Imperial, Manuel Kayser.

Actrices: Ana Adamuz (1. actriz), Carmen Collado, Durán, Matilde Galiana, La Peña, Dolores Vidal, María Vigo.

b) Repertorio:

El azar; El caudal de los hijos; Currito de la Cruz; Don Juan, buena persona; La mala hierba; La malquerida; Una mujer sin importancia; La pluma verde; El rayo; El último pecado.

1.3. Apareció esta actriz de nuevo en el escenario del teatro Bretón entre el 10 y el 13 de diciembre de 1926 al frente de la Gran compañía cómicodramática Adamuz-González.

a) Elenco:

Actores: Adolfo Benedicto, Escobar, Manuel González (1. ${ }^{\mathrm{er}}$ actor), Manuel Kayser.

Actrices: Ana Adamuz (1. ${ }^{a}$ actriz), Carmen Collado, Durán, María Vigo.

b) Repertorio:

Cristalina; El espanto de Toledo; ;Pero si yo soy mi hermano!; El sexo débil. 
1.4. Entre el 17 y el 23 de noviembre de 1927 la Compañía cómicodramática Adamuz-González actuó de nuevo en el logroñés teatro Bretón, dirigida por Manuel González ${ }^{1}$.

a) Elenco:

Actores: Adolfo Benedicto, Manuel González (1. ${ }^{\mathrm{er}}$ actor), Luis Guerrero, Casimiro Hurtado, Santiago Imperial, Arturo Marín, Fernando Sala.

Actrices: Ana Adamuz (1. actriz), María Armayor, Carmen Collado, María Cuevas, Matilde Galiana, Carmen Olmedo, Dolores Vidal.

b) Repertorio:

La caraba; El día menos pensado; La ermita, la fuente y el río; La hija de la Dolores; Los lagarteranos; La última novela.

1.5. Entre el 8 y el 15 de junio de 1929 volvió esta actriz al escenario del Bretón al frente de la Compañía cómico-dramática Adamuz-González.

a) Elenco:

Actores: Arturo Albert, Adolfo Benedicto, Vicente Burgos, Manuel González (1 er $^{\text {actor}), ~ L u i s ~ G . ~ G u e r r e r o, ~ C a s i m i r o ~ H u r t a d o, ~ S a n t i a g o ~ I m p e r i a l, ~}$ Manuel de Juan, José S. Luengo.

Actrices: Ana Adamuz (1. ${ }^{\mathrm{a}}$ actriz), Lucrecia Arnay, Matilde Galiana, M. ${ }^{\mathrm{a}}$ Lola Gómez, Consuelo Muñoz, Pilar Robles, Paz Robles, María Vigo, Carmen Villa.

b) Repertorio:

El alfiler; ;Béseme usted!; El caudal de los hijos; Los lagarteranos; No quiero, no quiero; Pepa Doncel; ¿Qué tienes en la mirada?; Sixto Sexto; El sofá, la radio, el peque y la hija de Palomeque; ;Usted es Ortiz!

1.6. La Gran compañía dramática Ana Adamuz se presentó en el teatro Bretón entre el 20 y el 25 de abril de 1946.

${ }^{1}$ Seguramente en todas las ocasiones en que el nombre de Manuel González aparece en la denominación de la compañía, éste actuaba como director, pero en las fuentes manejadas no lo especifica más que en esta ocasión; en la mayoría de los casos se da por hecho que el papel de director lo desempeñaba el primer actor. Este hecho se produce en más grupos, de modo que en lo sucesivo no lo comentaremos. 
a) Elenco:

Actores: Pedro Benedicto, Rafael Calvo (1. ${ }^{\mathrm{er}}$ actor), Carmelo Gandarias. Actrices: Ana Adamuz (1. ${ }^{\mathrm{a}}$ actriz), Lola Lemos, Consuelo Pastor, Josefina Robeda.

b) Repertorio:

Algo menos que una santa y algo más que una mujer; La condesa María; De muy buena familia; Madre Paz; La malquerida.

1.7. Denominando a su elenco Gran compañía de alta comedia Ana Adamuz actuó de nuevo esta artista en el escenario del Bretón logroñés, entre el 8 y el 12 de marzo de 1947.

a) Elenco:

Actores: Juan Beringola (1. ${ }^{\text {er }}$ actor).

Actrices: Ana Adamuz (1. ${ }^{\mathrm{a}}$ actriz).

b) Repertorio:

Angustias, la faraona; La ermita, la fuente y el río; Madre Paz; María, la viuda; La sin pecado.

1.8. La última actuación de esta actriz en Logroño durante el periodo estudiado se produjo en el mismo escenario, entre el 12 y el 15 de agosto de 1950 , al frente de la Gran compañía de alta comedia a la que da su nombre y que dirige Cecilio de Valcárcel.

a) Elenco:

Actores: José Carpena, José González, Valentín de Hojas, Ricardo Hurtado (1..$^{\mathrm{er}}$ actor), Francisco Linares Rivas, Luis Villar.

Actrices: Ana Adamuz (1. a actriz), Irene Barroso, Carmen de Lucio, Rosa M. ${ }^{a}$ Vega, Lola Villar.

b) Repertorio:

Los años perdidos; Celos del aire; Lo que hablan las mujeres; La noche del sábado. 


\section{COMPAÑÍA BASSÓ-NAVARRO}

2.1. La Compañía de Comedias Bassó-Navarro, dirigida por Nicolás Navarro, actuó por primera vez, durante el periodo analizado, entre el 24 y el 25 de noviembre de 1925, en el teatro Bretón de Logroño.

a) Elenco:

Actores: Nicolás Navarro (1. ${ }^{\mathrm{er}}$ actor).

Actrices: María Bassó (1. actriz).

b) Repertorio:

¡Señorita...!; La tonta del bote.

2.2. Entre el 9 y el 14 de febrero de 1927 volvió al escenario del Bretón esta compañía, que mantiene la misma denominación.

a) Elenco:

Actores: José Baviera, Pablo Bonell, Eusebio González, César Muro, Nicolás Navarro (1. ${ }^{\text {er }}$ actor), José Prado, José Valle.

Actrices: María Bassó (1. actriz), Carmen Morando, Pilar Pérez, Carmen Sánchez.

b) Repertorio:

Ángela María; La casa de los milagros; Charlestón; Doña Tufitos; La mano de Alicia; El matrimonio interino; Lo que ellas quieren; El señor cura y los ricos.

2.3. Bajo la denominación de Gran Compañía de Comedia Bassó-Navarro trabajaron estos actores de nuevo en el escenario del Bretón, entre el 14 y el 18 de noviembre de 1928.

a) Elenco:

Actores: Aurelio Castaños, Nicolás Navarro (1. ${ }^{\mathrm{er}}$ actor), Tino Rodríguez.

Actrices: María Bassó (1. actiz), Adela González, Carmen Morando. 
b) Repertorio:

Cuerdo amor, amo y señor; El negro que tenía el alma blanca; ;Pare usted la jaca, amigo!; ¿Quién te quiere a ti?

2.4. Apareció en el mismo escenario y con el mismo nombre esta compañía entre el 12 y el 15 de febrero de 1938.

a) Elenco:

Actores: Nicolás Navarro (1. ${ }^{\mathrm{er}}$ actor).

Actrices: María Bassó (1. actriz), Mercedes Pardo.

b) Repertorio:

Esta noche me emborracho; Los extremeños se tocan; Julieta compra un hijo; El negro que tenía el alma blanca.

2.5. Presentaron su trabajo de nuevo estos actores bajo la misma denominación en el teatro Bretón, entre 18 y el 20 de octubre de 1938.

a) Elenco:

Actores: Antonio Burgos, Nicolás Navarro (1..$^{\mathrm{er}}$ actor).

Actrices: María Bassó (1. actriz).

b) Repertorio:

El juramento de la Primorosa; La pimpinela escarlata; Susana tiene un secreto.

2.6. Otra vez en el Bretón, entre el 1 y el 6 de marzo de 1939, actuó de nuevo esta compañía.

a) Elenco:

Actores: Antonio Burgos, Rafael Catalán, Nicolás Navarro (1..$^{\text {er }}$ actor).

Actrices: María Bassó (1. a actriz), María Brieva, Pepita Martelo, Amalia Rodríguez. 
b) Repertorio:

El famoso Carballeira; Los hijos artificiales; Los hijos de la noche; El monje blanco; ¿Quién te quiere a ti?; La tonta del bote.

2.7. Entre el 7 y el 11 de noviembre de 1941 se presentó de nuevo esta compañía en el citado teatro logroñés.

a) Elenco:

Actores: Fernando Carrasco, Nicolás Navarro (1. ${ }^{\text {er }}$ actor), Luciano Ramallo, Adolfo del Río.

Actrices: María Bassó (1. a actriz), Patrocinio Hernández, M. ${ }^{a}$ Esperanza Navarro, Adriana Robles, Carmen Sanz, Ena Sedeño.

b) Repertorio:

El conflicto de Mercedes; Don Juan Tenorio; Hay que vivir; Topolino; Tortosa y Soler.

2.8. La Gran Compañía de Comedias Bassó-Navarro escenificó en otra ocasión en el teatro Bretón, entre el 9 y el 13 de noviembre de 1945.

a) Elenco:

Actores: José Granja, Nicolás Navarro (1. ${ }^{\mathrm{er}}$ actor), José Robles.

Actrices María Bassó (1. ${ }^{\mathrm{a}}$ actriz), Amparo Bori, M. ${ }^{\mathrm{a}}$ Luisa Marfil, Antonia Mas, M. ${ }^{a}$ Esperanza Navarro.

b) Repertorio:

La boda de papá; Los galeotes; Madame Pepita; ;Que familia!; ¡Tengo 17 años!

2.9. El mismo escenario volvieron a ocupar los actores de la Compañía de Comedia Bassó-Navarro entre el 17 y el 10 de octubre de 1946.

a) Elenco:

Actores: Manuel Escamilla., José Granja, Félix Navarro, Nicolás Navarro (1. ${ }^{\text {er }}$ actor), Miguel Pastor Mata. 
Actrices: María Bassó (1. ${ }^{\mathrm{a}}$ actriz), Amparo Conde, Charo Molina, M. ${ }^{\mathrm{a}}$ Esperanza Navarro, Paca Pons, Patrocinio Rico.

b) Repertorio:

La importancia de llamarse Ernesto; Mi casa es un infierno; Pigmalión; La romancera.

2.10. La última intervención de la compañía durante estos cincuenta años en Logroño, se produjo entre el 5 y el 7 de septiembre de 1947 en el mismo teatro Bretón y con la recuperación del adjetivo «gran» para la compañía.

a) Elenco:

Actores: José Granja, Julio Montijano, Nicolás Navarro (1. ${ }^{\mathrm{er}}$ actor), Miguel Pastor Mata.

Actrices: María Bassó (1. a actriz), Pepita Montoya, M. ${ }^{a}$ Esperanza Navarro.

b) Repertorio:

Un americano en Madrid; Esteban; La niña es un regalo.

\section{COMPAÑÍA DE FRANCISCO FUENTES}

3.1. La primera actuación de esta compañía durante los cincuenta años analizados tuvo lugar en el teatro Bretón, entre el 10 y el 28 de enero de 1902, con Francisco Fuentes como director.

a) Elenco:

Actores: Álamo, Fernando Altarriba, Julio del Cerro, Fauste, Francisco Fuentes (padre e hijo), La Riva, Llorente, Lliri, Rivelles.

Actrices: Antonia Arévalo, Guillén, Llorente, Mendizábal.

b) Repertorio:

El amigo; Amor salvaje; El bigote rubio; Los corridos; La criatura; El chiquillo; La dama de las camelias; De mala raza; Don Francisco de Quevedo; Un drama nuevo; Edmundo Kean; Electra; En el seno de la muerte; El estigma; Felipe Derblay; Hamlet; Los incasables; Juan José; Lanceros; La 
loca de la casa; El loco Dios; María del Carmen; La maya; Militares y paisanos; Los monigotes; Nicolás; El nido; Las personas decentes; El ratoncito Pérez; La reja; El sombrero de copa.

3.2. Esta compañía actuó de nuevo, en el mismo teatro, entre el 1 y el 5 de mayo de 1902.

a) Elenco:

Actores: Altarriba, Francisco Fuentes, La Riva, Llorente, Rivelles.

Actrices: Álamo, Antonia Arévalo, Guillén, Llorente.

b) Repertorio:

En el seno de la muerte; Hamlet; La praviana; Tratado de urbanidad; La vida es sueño; Las vírgenes locas.

3.3. La compañía, a cuyo frente figura como director Francisco Fuentes, volvió al escenario del Bretón entre el 19 y el 30 de septiembre de 1903.

a) Elenco:

Actores: Altarriba, Arévalo, Ricardo Calvo, Colom, Del Cerro, Francisco Fuentes.

Actrices: Abad, Antonia Arévalo, Cárcamo, Llorente, Monreal.

b) Repertorio:

Amor salvaje; Credulidad; De mala raza; El estigma; Felipe Derblay; El gran Galeoto; Hamlet; Los hijos artificiales; Lío-lío; Malas herencias; El marqués de Priola; Mi misma cara; Los miserables; El sombrero de copa; Los tocayos.

3.4. Bajo la denominación de Gran Compañía Cómico-dramática Francisco Fuentes actuó de nuevo, esta vez en el teatro Moderno, este grupo de actores, entre el 18 y el 30 de septiembre de 1913.

a) Elenco:

Actores: Luis Alcaide, Fernando Altarriba, Cristino Álvarez, Emilio Arévalo, Pedro Barinaga, Ángel Cores, Francisco Fuentes (padre e hijo), Ma- 
cías, Enrique Mandredi, Miguel Pozanco, Nicolás Rodríguez, José Soto, Germán Sylas.

Actrices: María Anaya, Antonia Arévalo (1. a actriz), Julia Artola, Elena Fernández, Carmen Manfredi, Rafaela Montoya, Carmen Muñoz, Juana Robles, Adelina Rodríguez, Matilde Rodríguez, Elvira Rojas, Encarnación Ruiz Blanco, Guillermina Soto, Concepción Villar.

b) Repertorio:

La buena voluntad; Camino adelante; La comida de las fieras; Divorciémonos; La Dolores; El estigma; La estirpe de Júpiter; Los hijos artificiales; Los hugonotes; Los intereses creados; El ladrón; Madame Pepita; Malvaloca; Más fuerte que el amor; Un negocio de oro; El nido ajeno; La noche del sábado; El octavo no mentir; La princesa Bebé; Sobrevivirse; Tierra baja.

3.5. De nuevo en el escenario del Moderno presentó su trabajo la Compañía Cómico-dramática Francisco Fuentes entre el 20 de diciembre de 1913 y el 6 de enero de 1914.

a) Elenco:

Actores: Luis Alcaide, Fernando Altarriba, Cristino Álvarez, Emilio Arévalo, Pedro Barinaga, Ángel Cores, Francisco Fuentes (padre e hijo), Enrique Manfredi, Carlos de Nicolás, Ignacio Ortega, Miguel Pozanco, Nicolás Rodríguez, José Soto, Germán de Sylas.

Actrices: María Anaya, Antonia Arévalo, Julia Artola, Concepción Castañeda, Elena Fernández, Carmen Illescas, Carmen Manfredi, Rafaela Montoya, Juana Robles, Adelina Rodríguez, Elvira Rojas, Encarnación Ruiz, Guillermina Soto, Juana Vila, Concepción Villar.

b) Repertorio:

El abuelo; El amor a oscuras; Cyrano de Bergerac; Divorciémonos; La Dolores; Don Juan Tenorio; En cuarto creciente; El estigma; Felipe Derblay; El gran Galeoto; Hamlet; Los hijos artificiales; Juan José; El ladrón; El lobo; La loca de la casa; El loco Dios; La malquerida; Malvaloca; El matrimonio interino; La muñeca trágica; La noche del sábado; Los pastores; Traidor, inconfeso y mártir.

3.6. Recuperó el escenario del teatro Bretón la Compañía de Comedias de Francisco Fuentes entre el 11 y el 15 de noviembre de 1922. 
a) Elenco:

Actores: Francisco Fuentes (padre e hijo), Ricardo Vargas.

Actrices: Joaquina Almarches, Társila Criado, Dolores Ranero.

b) Repertorio:

Los caballos negros; Constantino Plá; Lluvia de hijos; El misterio del cuarto amarillo; El pleito; La señorita Ángeles; ;360 mujeres!

3.7. Esta vez, bajo la denominación de Compañía cómico-dramática Francisco Fuentes, volvió a efectuar representaciones, en el teatro Bretón, entre el 22 de diciembre de 1923 y el 6 de enero de 1924.

a) Elenco:

Actores: Francisco Fuentes (padre e hijo).

Actrices: Társila Criado (1. a actriz), Julia Sala.

b) Repertorio:

Las alas rotas; La copa del olvido; Las cosas de Gómez; Cristalina; El cuarto de gallina; Currito de la Cruz; La dama de las camelias; Es mi hombre; El ladrón; Lluvia de hijos; ;Mala madre!; La malquerida; Malvaloca; El orgullo de Albacete; La pena de los viejos; La propia estimación; Santa Isabel de Ceres; La señorita Ángeles; Traidor, inconfeso y mártir; El tren rápido; Yo amo, tú amas.

\section{COMPAÑÍA LADRÓN DE GUEVARA}

4.1. Actuó por primera vez esta actriz en Logroño, durante estos cincuenta años analizados, entre el 13 y el 16 de noviembre de 1923; lo hizo al frente de la Compañía Cómico-dramática Ladrón de Guevara-Rivelles y en el escenario del teatro Bretón.

a) Elenco:

Actores: Juan de Orduña, Fernando Porredón (1. ${ }^{\text {er }}$ actor cómico), M. Rivas, Rafael Rivelles (1. ${ }^{\text {er }}$ actor), M. Rodríguez, Enrique Vilches. 
Actrices: Almudena Ayala, Juana Cáceres, Laura Cámara, Pilar Cebrián, Carmen Collado, Encarna Gutiérrez, M. ${ }^{\mathrm{a}}$ Fernanda Ladrón de Guevara (1. ${ }^{\mathrm{a}}$ actriz), Matilde Rodríguez, Josefina Satorres, M. ${ }^{a}$ Ofelia Zapico.

b) Repertorio:

Al rugir el león; El chiquillo; La loca aventurera; El madrigal de la cumbre; Ramo de locura.

4.2. Volvió la compañía Ladrón de Guevara-Rivelles al teatro Bretón entre el 18 y el 21 de noviembre de 1924.

a) Elenco:

Actores: Fernando Porredón (1. ${ }^{\text {er }}$ actor cómico), Rafael Rivelles (1. ${ }^{\text {er }}$ actor).

Actrices: M. ${ }^{\mathrm{a}}$ Fernanda Ladrón de Guevara (1. ${ }^{\mathrm{a}}$ actriz).

b) Repertorio:

Aire de fuera; El chiquillo; De mala raza; Felipe Derblay; Rirri.

4.3. De nuevo en el Bretón pudo ver el público logroñés a la Compañía de Comedias Ladrón de Guevara-Rivelles, entre el 16 y el 18 de febrero de 1927.

a) Elenco:

Actores: Julio F. Alyman, Evaus, José Portes, Rafael Rivelles (1. ${ }^{\text {er }}$ actor), Francisco, Urquijo, Gonzalo Vico.

Actrices: Ayala, Juana Cáceres, Adela Carboné, M. ${ }^{a}$ Fernanda Ladrón de Guevara, Josefina Lamas, Elena Salvador.

b) Repertorio:

La gran duquesa y el camarero; Un hombre y una mujer; La mariposa que voló sobre el mar.

4.4. Con la misma denominación volvió a actuar esta compañía, en el mismo escenario, entre el 24 y el 27 de abril de 1934. 
a) Elenco:

Actores: Fernández de Córdoba, Ramiro de la Mata, José Portas, Rafael Rivelles, Emilio Thuillier (1 ${ }^{\text {er }}$ actor).

Actrices: M. ${ }^{\mathrm{a}}$ Fernanda Ladrón de Guevara.

b) Repertorio:

Amores y amoríos; La mujer X; El otro amor; El pan comido en la mano; El rapto de Elena.

4.5. De nuevo en el Bretón, entre el 24 y el 30 de septiembre de 1934, ofreció su trabajo este elenco, con la misma denominación anterior.

a) Elenco:

Actores: Francisco Alarcón, José Blanch, Benito Cobeña, Manuel Luna, Adolfo del Río, Rafael Rivelles, Manuel Soto, Francisco Urquijo, Jesús Valero. Actrices: M. ${ }^{\mathrm{a}}$ Fernanda Ladrón de Guevara.

b) Repertorio:

La divina comedia; Felipe Dreblay; El gran Galeoto; La mujer X; El nido ajeno; La paz de Dios; El río dormido; Yo quiero ser diputado.

4.6. La ahora Gran Compañía de Comedias Ladrón de Guevara, dirigida por Manuel González, actuó entre el 31 de mayo y el 2 de junio de 1939 en el escenario del teatro Bretón.

a) Elenco:

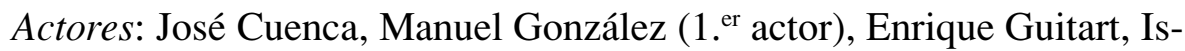
mael Merlo, Alejandro Noya, Miguel Pozanco.

Actrices: Consuelo Company, Maruja García Alonso, Luisa Jerez, M. ${ }^{\mathrm{a}}$ Fernanda Ladrón de Guevara.

b) Repertorio:

El compañero Pérez; Poca cosa es un hombre; La señorita Ángeles.

4.7. Bajo la misma denominación de la compañía volvió esta actriz al teatro Bretón logroñés entre el 27 y el 30 de noviembre de 1939. 
a) Elenco:

Actores: Enrique Guitart, Ismael Merlo, Alejandro Noya.

Actrices: Consuelo Company, Maruja García Alonso, M. ${ }^{a}$ Fernanda Ladrón de Guevara, Amparo Rivelles.

b) Repertorio:

Amores y amoríos; La madre guapa; La morocha; La papirusa.

4.8. Entre el 14 y el 16 de diciembre de 1939 la misma compañía efectuó nuevas representaciones en el mismo teatro Bretón.

a) Elenco:

Actores: Pedro Gimier, Enrique Guitart, Miguel de Llano, Fernando Marín, Ismael Merlo, Alejandro Noya, Manuel de Velasco.

Actrices: Pilar Bienest, Consuelo Company, M. ${ }^{a}$ Luisa Corominas, Maruja García Alonso, M. ${ }^{\mathrm{a}}$ Fernanda Ladrón de Guevara, M. ${ }^{\mathrm{a}}$ Luisa Ponte, Amparo Rivelles.

b) Repertorio:

Las colegialas; El secreto de la mujer X; Siete mujeres.

4.9. En esta ocasión, dirigida por Pedro Larrañaga, esta misma compañía actuó en el mismo escenario entre el 18 y el 23 de septiembre de 1942.

a) Elenco:

Actores: Alfonso de Córdoba, Félix Dafauce, Pedro Gil, Pedro Larrañaga, Miguel de Llano.

Actrices: Laura Alcoriza, Consuelo Company, M. ${ }^{a}$ Fernanda Ladrón de Guevara.

b) Repertorio:

Chiruca; La enemiga; La honradez de la cerradura; Malvaloca; María Antonieta; La papirusa.

4.10. La última visita a la ciudad de Logroño de esta actriz durante el periodo analizado tuvo lugar entre el 30 de diciembre de 1948 y el 4 de enero 
de 1949, al frente de la Gran Compañía de Alta Comedia Ladrón de Guevara, en el teatro Bretón.

a) Elenco:

Actores: Antonio Armet, Julio Miguel, Manuel de Sabatini.

Actrices: Luisa Díaz de Velasco, Cecilio Gordón, M. ${ }^{a}$ Carmen Gordón, M. ${ }^{a}$ Fernanda Ladrón de Guevara, Pepa Martín, Ana de Siria.

b) Repertorio:

Cancela; La enlutada; El ladrón; Mujer; No tiene corazón; Sabela de Cambados.

\section{COMPAÑÍA LÓPEZ HEREDIA}

5.1. La primera vez que esta actriz actuó en Logroño fue en el teatro Bretón, entre el $10 \mathrm{y}$ el 13 de enero de 1928. Como director del elenco, denominado Compañía de Comedia Irene López Heredia, figuraba Ricardo Baeza.

a) Elenco:

Actores: Juan Espantaleón, José Bruguera, Antonio Vico, Ernesto Vilches. Actrices: Ana M. ${ }^{a}$ Custodio, Irene López Heredia, Carmen Nieto, Herminia Peñaranda, Dolores Valero.

b) Repertorio:

El corazón manda; Lady Frederick; Un marido ideal; Tambor y cascabel.

5.2. Entre los días 7 y 10 de marzo de 1936, volvió esta artista al escenario del Bretón al frente de la Compañía de Comedia López Heredia-Asquerino.

a) Elenco:

Actores: Gabriel Algara, Antonio Armet, Mariano Asquerino, José Guijarro, Francisco Hernández, Gonzalo Llorens, Ricardo Vargas.

Actrices: Carmen León, Irene López Heredia, Carmen López Lagar, Pascuala Mesa, Isabel Ortega, Isabel Pallarés. 
b) Repertorio:

El báculo y el paraguas; La dama del antifaz; La inglesa sevillana; La papirusa.

5.3. En la siguiente visita a nuestra ciudad, de nuevo en el Bretón, este elenco se denomina Gran Compañía de Alta Comedia López Heredia-Asquerino; actuó entre el 24 y el 27 de octubre de 1939.

a) Elenco:

Actores: Mariano Asquerino, José Blanch, Federico Górriz, José Latorre, José Montijano, Luis Porredón, Ricardo Vargas.

Actrices: Adela Carboné, Irene López Heredia, Asunción Montijano, Concha Montijano, Isabel Ortega, Consuelo Pallarés, M. ${ }^{a}$ Isabel Pallarés, Ana de Siria, Antonia Tejedor.

b) Repertorio:

Campo de armiño; La inglesa sevillana; El río dormido; Suspenso en amor.

5.4. Como Compañía de Comedia López Heredia-Asquerino volvió este grupo de artistas al escenario anterior, entre el 31 de octubre y el 6 de noviembre de 1941.

a) Elenco:

Actores: Rafael Alonso, Mariano Asquerino, José Bernal, Rafael Calvo, Federico Górriz, Pedro Hurtado, Juan Monfort, José Montijano.

Actrices: Adela Carboné, Amelia García, Irene López Heredia, Pascuala Mesa, Asunción Montijano, M. ${ }^{a}$ Antonia Tejedor.

b) Repertorio:

El collar de estrellas; Escuela de princesas; Lo cursi; Una mujer sin importancia; La noche del sábado; La princesa Bebé; Rosas de otoño.

5.5. De nuevo la actriz apareció sola al frente de la Compañía de Comedia López Heredia; actuó en el Bretón entre el 16 y el 22 de enero de 1943. 
a) Elenco:

Actores: José Bernal, Luis Calderón, Emilio C. Espinosa, José Montijano, Fulgencio Nogueras.

Actrices: Matilde Guarnerio, Irene López Heredia, Ana M. ${ }^{a}$ Méndez, Pascuala Mesa, Josefina Palacios.

b) Repertorio:

Ha entrado una mujer; La inglesa sevillana; Lady Amarilla; La madre guapa; Pero la vida no es sueño; El rosario; Suspenso en amor.

5.6. Como Gran Compañía de Alta Comedia López Heredia volvió este grupo al mismo escenario logroñés, entre el 1 y el 7 de septiembre de 1944.

a) Elenco:

Actores: Emilio C. Espinosa.

Actrices: Irene López Heredia.

b) Repertorio:

Adriana; La carta; La condesa Butterfly; Lady Frederick; Madame Cecilia «Modas»; María Victoria; La sombra.

5.7. De nuevo en el Bretón, entre el 13 y el 16 de septiembre de 1945, presentó su trabajo la Compañía de Comedia López Heredia.

a) Elenco:

Actores: José Bernal, José Bruguera, Vicente Soler.

Actrices: Carmen Blázquez, Irene López Heredia, Asunción Montijano, M. ${ }^{a}$ Luisa Tejedor.

b) Repertorio:

Las flores; Un marido ideal; La mujer de nadie; Ventolera.

5.8. Volvió esta actriz a actuar en Logroño al frente de la Gran Compañía de Alta Comedia López Heredia; las funciones se realizaron en el Bretón entre el 21 y el 24 de agosto de 1947. 
a) Elenco:

Actores: José Bernal, Manuel Díaz de Velasco, Antonio Prieto, Luis Torrecilla.

Actrices: Carmen Blázquez, Montserrat Casas, Lois Gómez, Irene López Heredia, Asunción Montijano, M. ${ }^{a}$ Rosario Soriano.

b) Repertorio:

La infanzona; Miedo; Titania.

5.9. La Compañía de Comedia López Heredia ofreció una serie de funciones, en el teatro anterior, del 26 al 29 de agosto de 1948.

a) Elenco:

Actores: José Bernal, Luis Durán, Llano, Antonio Prieto.

Actrices: Carmen Blázquez, Montserrat Casas, Mary González, Irene López Heredia, Asunción Montijano.

b) Repertorio:

Así son todas; Campo de armiño; El mayor pecado; El placer de los dioses; El rival de su mujer.

5.10. La última visita a la capital riojana, durante este periodo, de la Gran Compañía de Comedia López Heredia se realizó entre el 1 y el 5 de noviembre de 1950, en el teatro Bretón. Como director artístico llevaba a Luis Hurtado Álvarez.

a) Elenco:

Actores: Francisco Alonso, Manuel Díaz de Velasco, Luis García Ortega, Antonio Queipo.

Actrices: Carmen Blázquez, M. ${ }^{a}$ Angélica Domingo, Mary González, Irene López Heredia, Asunción Montijano, Marta Rotger.

b) Repertorio:

Divorcio de almas; El fiscal y la acusada; Una mujer sin importancia; Por el camino de la vida; Rosas de otoño. 


\section{COMPAÑÍA MARTÍ-PIERRÁ}

6.1. La Gran Compañía de Comedia Martí-Pierrá actuó por primera vez en Logroño, durante el periodo estudiado, entre el 6 y el 13 de noviembre de 1928, en el teatro Bretón.

a) Elenco:

Actores: Joaquín Fernández Roz, José Morcillo, Francisco Pierrá, Francisco Puyol, Modesto Rivas.

Actrices: Milagros Andrés, Josefina Lamas, Matilde López Roldán, Mercedes L. Manero, Amparo Martí, Concepción Villar.

b) Repertorio:

Caridad; Los dos tronos; Hay que vivir; Lola y Loló; Mimí Valdés; La muralla de oro; El rosal de las tres rosas; Señor... ¿por qué son tan guapas?

6.2. Esta vez sin el adjetivo «gran», actuó de nuevo en el Bretón esta compañía entre el 8 y el 1 de enero de 1929.

a) Elenco:

Actores: Joaquín Fernández Roa, Luis Morcillo, Francisco Pierrá.

Actrices: Josefina Lamas, Matilde López Roldan, Amparo Martí.

b) Repertorio:

El automóvil del rey; La boda de Quinita Flores; Mi hermana Genoveva; ¡Te quiero, te adoro!

6.3. Con igual denominación volvió este elenco al mismo escenario logroñés, entre el 22 de diciembre de 1929 y el 6 de enero de 1930.

a) Elenco:

Actores: Juan Aguado, Alcaine, Félix Dafauce, Enrique A. Diosdado, Joaquín Fernández Roa, Luis Morcillo, Osete, Francisco Pierrá, Francisco Puyol, Gonzalo Vico.

Actrices: Amparo Martí. 
b) Repertorio:

El alfiler; ;Atrévete, Susana!; Cien comedias y un drama; Cuento de hadas; Las grandes fortunas; El ladrón; Lola y Loló; Los mosquitos; Los muñecos; La muralla de oro; Para ti es el mundo; ¡Pégame, Luciano!; El tío Morris; El último lord.

6.4. Volvió al Bretón esta misma compañía, actuando entre el 31 de octubre y el 11 de noviembre de 1931.

a) Elenco:

Actores: Juan Aguado, Félix Dafauce, Santiago Imperial, José Morcillo, Pablo Muñiz, Francisco Pierrá, Francisco Puyol.

Actrices: Pilar Calvo, Concepción Farfán, Teresa Gamborino, Amparo Martí, Carmen V. Palencia, M. ${ }^{a}$ Victoria Rivera, Concepción Villar.

b) Repertorio:

Don Juan Tenorio; ;Esta noche me emborracho!; ;Este hombre me gusta!; ¡Pecar... hacer penitencia!; ;Pégame, Luciano!; ;Qué gran chico!; Rata de hotel; ¡Te quiero, te adoro!; La última aventura; El último lord.

6.5. De nuevo como Gran Compañía de Comedia Martí-Pierrá, se presentó en el mismo escenario este grupo de actores entre el 19 y el 21 de mayo de 1933.

a) Elenco:

Actores: Juan Aguado, Chavarri, Félix Dafauce, José Morcillo, Pablo Muñiz, Francisco Pierrá, Francisco Puyol.

Actrices: Pilar Calvo, Concepción Farfán, Amparo Martí, Carmen V. Palencia, Magdalena Roger, Carmen Sabat, Concepción Villar.

b) Repertorio:

El corazón ciego; Escuela de millonarias; María, la famosa.

6.6. Entre el 2 y el 5 de enero de 1934 volvió esta compañía a presentar su trabajo en el escenario del Bretón logroñés. 
a) Elenco:

Actores: Juan Aguado, Julio Francia, José Morcillo, Pablo Muñiz, Francisco Pierrá, Del Valle.

Actrices: Pilar Calvo, Concepción Farfán, Amparo Martí, Carmen V. Palencia, Concepción Villar.

b) Repertorio:

Como tú ninguna; La chica de Buenos Aires; Los hijos de la noche; El hombre de confianza.

6.7. La siguiente actuación de esta compañía tuvo lugar entre el 4 y el 11 de noviembre de 1940, otra vez en el Bretón.

a) Elenco:

Actores: Miguel Pastor Mata, Fernando Montenegro, José Montijano, Adrián Ortega, Francisco Pierrá, José Robles.

Actrices: Pilar Calvo, Concha Farfán, Carita Marinas, Amparo Martí, Josefina Roca.

b) Repertorio:

El derecho de los hijos; Eloísa está debajo de un almendro; En poder de Barba Azul; La florista de la reina; Lo increíble; Morena clara; La otra honra.

6.8. El escenario del Bretón fue de nuevo el elegido por esta compañía para realizar sus actuaciones, entre el 25 de septiembre y el 1 de octubre de 1941; en esta ocasión se cita como director a Francisco Pierrá.

a) Elenco:

Actores: Fernando Montenegro, Miguel Pastor Mata, Francisco Pierrá. Actrices: Concha Farfán, Amparo Martí, Josefina Roca.

b) Repertorio:

Atrévete, Susana; La boda de Quinita Flores; La casa de modas; Madrinita buena; Los muñecos; Un timbre que no suena; El último lord. 
6.9. Entre el 15 y el 22 de octubre de 1942 volvió a visitar Logroño esta compañía; pusieron en escena sus obras en el teatro Bretón.

a) Elenco:

Actores: José Balaguer, Antonio Garisa, Francisco Pierrá, Antonio Queipo, Adolfo del Río.

Actrices: M. ${ }^{a}$ Luisa Amado, Pilar Calvo, Carita Marinas, Amparo Martí, Josefina Roca.

b) Repertorio:

La campeón; Canción de Navidad; Juanita Torbellino; Lo increíble; Plenos poderes; El rigodón del amor; La señorita Suspenso.

6.10. Regresó esta compañía al mismo coliseo logroñés entre el 21 de septiembre y el 1 de octubre de 1943.

a) Elenco:

Actores: Francisco Pierrá.

Actrices: Amparo Martí.

b) Repertorio:

El azahar de la novia; Juanita Torbellino; Una mujer elegante; Nidos sin pájaros; Primero vivir; La raza; La señorita está loca; El último lord.

6.11. De nuevo el Bretón volvió a ser testigo del trabajo de esta compañía, que volvió al mismo entre el 17 y el 23 de noviembre de 1944.

a) Elenco:

Actores: Francisco Pierrá.

Actrices: Amparo Martí.

b) Repertorio:

Aquella mujer...; Mujeres modernas; La otra honra; Paulina se escapa; La señorita Suspenso; Solita ya no está sola. 
6.12. Actuó otra vez esta compañía en el mismo escenario entre el 28 de noviembre y el 4 de diciembre de 1945.

a) Elenco:

Actores: José Balaguer, Julio Gorostegui, Francisco Pierrá.

Actrices: Concha Farfán, Amparo Martí, M. ${ }^{a}$ Luisa Ponte.

b) Repertorio:

Dora (la espía); Gran mundo; He comprado una mujer; Mi hija será feliz; Pasar por la vida; El último lord.

6.13. Finalizaron estos actores la serie de visitas realizadas a Logroño durante estos cincuenta años con una serie de obras, de nuevo en el Bretón. Las funciones se efectuaron entre el 3 y el 9 de diciembre de 1947.

a) Elenco:

Actores: Gregorio Alonso, José Balaguer, Francisco Faure, León Lavalle, Julio Montijano, Francisco Pierrá.

Actrices: Concha Farfán, Margarita García Ortega, Amparo Martí, Emma Picot.

b) Repertorio:

Las cruces; Una gallega en Nueva York; Hay que matar al difunto; La otra honra; La prueba del ángel; La señorita está loca; El último lord.

\section{COMPAÑía JOSÉ MONTIJANO}

7.1. La primera visita que durante el siglo XX realizó este actor a Logroño tuvo lugar entre el 18 y el 27 de diciembre de 1903, para representar una serie de obras en el teatro Bretón.

a) Elenco:

Actores: Écija, Fonseca, Lacao, López Portillo, José Montijano, Mula, Sánchez.

Actrices: Berenguer, Bueno, Hortensia Egea, García Muñoz, Mercedes Martín, Salá. 
b) Repertorio:

La aldea de San Lorenzo; Las codornices; La cuerda floja; Los dos pilletes; Entre doctores; Mariucha; La pasionaria; Los pobres de Madrid; El soldado de San Marcial; Tortosa y Soler; La vida de un jugador.

7.2. Bajo la denominación de Compañía Cómico-dramática Montijano volvió este grupo al escenario anterior, entre el 13 de marzo y el 18 de abril de 1909, con un breve descanso de una semana, del 5 al 10 de abril.

a) Elenco:

Actores: Capilla, Écija, Martínez, José Montijano, José Montijano (hijo), Ortega, Pedrosa, Ríos, Vallarino.

Actrices: Abrines, García, Montijano, Núñez, Ortega, Ríos, Sanz.

b) Repertorio:

El abuelo; A la luz de la luna; El amor que pasa; Bodas de plata; La cizaña; Las codornices; Con plumas de pavo real; La cuerda floja; Las de Caín; La Dolores; Los dos pilletes; La escondida senda; Floriana; Francfort; La fuerza bruta; González y González; Hija única; Los hijos artificiales; Los hugonotes; El incierto porvenir; Los intereses creados; ;Lo que vale el talento!; Mancha que limpia; Mariana; El matrimonio interino; Mi cara mitad; El nido; La pasionaria; Por las nubes; La primera postura; Raffles; El señor de Bobadilla; Sherlock Holmes; El soldado de San Marcial; El sombrero de copa.

7.3. Bajo la misma denominación de su compañía volvió a actuar este actor, en el principal coliseo logroñés, entre el 18 de diciembre de 1909 y el 10 de enero de 1910.

\section{a) Elenco:}

Actores: Ricardo Capilla, Enrique Écija, Joaquín Fernández Roa, Manuel Lozano, Celedonio Martínez, José Montijano (padre e hijo), Antonio Pedrosa, Ramón Vallarino.

Actrices: Elena Gil López, Josefa Luján, Consuelo Montijano, Asunción Núñez, Pilar Ortega, Concepción Ríos, Concepción Sanz. 
b) Repertorio:

El abuelo; El afinador; El amor que pasa; Bodas de plata; La careta verde; La cizaña; Las codornices; Los chorros del oro; La escuela de las princesas; La fuerza bruta; Los gansos del Capitolio; El genio alegre; El gran tacaño; Los hugonotes; Los intereses creados; Lo cursi; Los malhechores del bien; Mariana; El matrimonio interino; El místico; El nido; El oso muerto; El padrón municipal; Por las nubes; Por los suelos; Las tres máscaras; Zaragüeta.

7.4. De nuevo en el Bretón, actuó la ahora Compañía de Comedia Montijano entre el 17 de marzo y el 1 de abril de 1917.

a) Elenco:

Actores: Ricardo Canales, Manuel Capilla, Fernando de Castro, Alfredo Corcuera, Ricardo Capilla, Luis Jiménez, José Montijano (padre e hijo), José Ramírez, Alberto Serrano, Ramón Vallarino.

Actrices: Almudena Ayala, Elvira Bernáldez, Carmen Caballero, Concepción García, Eloísa Martínez, Consuelo Montijano, Asunción Núñez, Amelia Ordóñez, Luisa Zamora, Leonor Zugasti.

b) Repertorio:

El cardenal; La chocolaterita; En el limpio solar; El gran Galeoto; Lo que vale el talento; El príncipe Juanón; La propia estimación; La señorita de Trevélez.

7.5. La nombrada esta vez Compañía de Comedia y Drama José Montijano ofreció funciones al público logroñés de nuevo; se realizaron en el Bretón entre el 14 y el 26 de marzo de 1918.

a) Elenco:

Actores: Ricardo Capilla, Víctor García Navarro, Federico Iglesias, Luis Jiménez, José Montijano (padre e hijo), Marcial Navarro, Octavio Prieto, Alberto Serrano, Ramón Vallarino.

Actrices: Emilia Casanova, Presentación Espallardo, Isabel Garcés, Asunción M. García, Concepción García, Soledad Martínez, Consuelo Montijano, Asunción Núñez, Laura Ramírez, Irene Ríos. 
b) Repertorio:

El abuelo; Así se escribe la historia; Los cachorros; Como hormigas; Francfort; La loca de la casa; Madame Pepita; Nicolás; Pipiola; El rayo; El roble de la Jarosa.

7.6. Bajo la misma denominación realizó una serie de puestas en escena este grupo de actores, de nuevo en el teatro Bretón. Fue entre el 7 de diciembre de 1918 y el 10 de enero de 1919.

a) Elenco:

Actores: José Bernáldez, Manuel Capilla, Ricardo Capilla, José Encinas, Luis Jiménez, Carlos Lanzarote, José Montijano (padre e hijo), Octavio Prieto, Alberto Serrano, Ramón Vallarino.

Actrices: Carmen Cebrián, Asunción M. García, Concepción García Sanz, Soledad Martínez, Consuelo Montijano, Asunción Núñez, Amelia Ordóñez, Irene Ríos, Dora Sancho, Enriqueta Sancho, Luisa Sancho.

b) Repertorio:

¡Adiós, juventud!; Amores y amoríos; La barba de Carrillo; Los campesinos; La carcajada o amor de hijo; El cardenal; La cuerda floja; Don Juan Tenorio; Doña Clarines; Los dos pilletes; En cuerpo y alma; En Flandes se ha puesto el sol; Esclavitud; Fantasmas; La frescura de Lafuente; La garra; El infierno; La Inmaculada de los Dolores; Los intereses creados; Juan José; Marianela; Mister Beverley; El místico; Un negocio de oro; Nicolás; Los ojos de luto; Porque sí; El príncipe Juanón; Puebla de las mujeres; Que viene mi marido; Ruido de campanas; La señorita de Trevélez; El soldado de San Marcial; Traidor, inconfeso y mártir; El verdugo de Sevilla.

7.7. Volvió a actuar en el teatro Bretón esta compañía entre el 19 y el 29 de abril de 1919.

a) Elenco:

Actores: Capilla, Graci, José Montijano (hijo), Ordóñez, Alberto Serrano. Actrices: Consuelo Montijano, Dora Sancho, Enriqueta Sancho. 
b) Repertorio:

La casa de la Troya; Cobardías; La chocolaterita; Los dos pilletes; Un drama de Calderón; Esclavitud; Pipiola; Porque sí; Los semidioses; La señorita está loca; El sueño dorado.

\section{COMPAÑÍA DE FRANCISCO MORANO}

8.1. La primera aparición de este actor en Logroño en la época estudiada se produjo en el año 1904; el elenco respondía al nombre de Compañía Moreno-Morano y realizó sus funciones en el teatro Bretón entre el 21 de septiembre y el 5 de octubre de 1904.

a) Elenco:

Actores: Francisco Morano, Nieva, Norro, Porredón, Vázquez.

Actrices: Bagá, Nieves Gómez, Martínez, Matilde Moreno.

b) Repertorio:

Aire de fuera; El amigo; Una broma pesada; La castellana; El crío; ¡Deshonor!; La dicha ajena; Don Álvaro o la fuerza del sino; Entre doctores; El estigma; Fedora; La fierecilla domada; El flechazo; Las flores; Nubecilla de verano; Otelo; Tortosa y Soler; La Tosca.

8.2. Reapareció este actor de nuevo en el Bretón al frente de la Compañía Cobeña-Morano, dirigida por Federico Oliver; ofrecieron una serie de obras entre el 9 y el 17 de mayo de 1908.

a) Elenco:

Actores: Benito Cobeña, Francisco Comes, Manuel Domínguez, Federico Llorens, Ricardo Manso, Francisco Morano (1 ${ }^{\text {er }}$ actor.), Manuel Perrín, Agustín Povedano, Rafael Requena, Leovigildo Ruiz Tatay, Carlos Soto.

Actrices: M. ${ }^{a}$ Luisa Ahijón, Josefina Álvarez, María Anaya, Carmen Cobeña (1. ${ }^{a}$ actriz), Josefina Cobeña, Consuelo Izaguirre, Isabel Luna, Matilde Lorente, Dolores Soriano, Ángela Tamames, Mercedes Villabona.

b) Repertorio:

Los amantes de Teruel; Carambolas; Un drama en cinco minutos; Flaquezas humanas; El genio alegre; Lealtad de una mujer y aventuras de una 
noche; La montaraza de Olmeda; Los ojos de los muertos; O locura o santidad; Papá Lebonnard; La pesca del millón; El preceptor; Señora ama.

8.3. La Compañía dramática, dirigida por Francisco Morano, regresó en 1912 para actuar en el teatro Bretón. Lo hizo entre los días 28 de febrero y 12 de marzo.

a) Elenco:

Actores: Ernesto Álvarez, Nicolás Díaz-Perchicot, Manuel Gutiérrez, Manuel Kayser, José María, Francisco Molinero, Francisco Morano, Víctor Pastor, Gerardo Peña, Patrico Redondo, Adolfo H. del Río.

Actrices: Josefina Álvarez Segura, María Antón, Cruz Armiñana, Esperanza Bedoya, Amparo Fernández Villegas, Pura Fernández Villegas, Amelia Sánchez, Milagros Texeiro, María Santoncha, Elisa Villa.

b) Repertorio:

El alcalde de Zalamea; La alquería; Amor salvaje; Amo y criado; El aventurero; La cena de la burlas; El centenario; La chocolaterita; El drama de la vida; En Flandes se ha puesto el sol; Fedora; Lo cursi; La luna de la sierra; El místico; Papá Lebonnard; Primavera en otoño; La raza; Sangre gorda; Traidor, inconfeso y mártir.

8.4. Con el mismo nombre de compañía y de nuevo como director volvió de nuevo Francisco Morano a las tablas del Bretón; fue entre el 20 de septiembre y el 10 de octubre de 1912.

a) Elenco:

Actores: Ernesto Álvarez, Joaquín Campos, Nicolás Díaz-Perchichot, Luis Domínguez, Antonio Estévez, José Marín, Francisco Morano, Víctor Pastor, Fernando Porredón (1. ${ }^{\text {er }}$ actor), Patricio Redondo, Adolfo H. del Río, Carlos Soto.

Actrices: Cruz Almiñana, María Antón, Esperanza Bedoya, Carmen Cepillo, Cecilia Delaga, Amparo Fernández Villegas (1. actriz), Pura Fernández Villegas, Elena Rodríguez, Amalia Sánchez, Ascensión Vivero.

b) Repertorio:

El alcalde de Zalamea; El ama de la casa; Amor y ciencia; El barón de Tronco Verde; Bodas de plata; La carcajada; El centenario; La cuerda floja; 
La chocolaterita; De mala raza; Don Álvaro o la fuerza del sino; Doña Clarines; En el teléfono; En Flandes se ha puesto el sol; Entre doctores; El estigma; Felipe Derblay; La fierecilla domada; Las flores; El gran Galeoto; Juan José; El ladrón; Lo cursi; La losa de los sueños; Malvaloca; La muerte civil; Papá Lebonnard; Pepa la frescachona o el colegial desenvuelto; Por la nubes; Puebla de las mujeres; El sexo débil; La Tosca.

8.5. La Compañía Morano actuó, en el Bretón de nuevo, entre el 7 y el 15 de mayo de 1913, bajo la dirección de Francisco Morano.

\section{a) Elenco:}

Actores: Ernesto Álvarez, Joaquín Campos, Nicolás Díaz-Perchicot, Jenaro Guillot, José Marín, Francisco Morano (1. er actor), Víctor Pastor, Emilio Portes, Patricio Redondo, Carlos Soto.

Actrices: Luisa Alcalá, Cruz Almiñana, María Antón, Esperanza Bedoya, Carmen Cepillo, Amparo Fernández Villegas (1. actriz), Pura Fernández Villegas, Elena Rodríguez, Amalia Sánchez, Ramona Valdivia.

b) Repertorio:

Amor salvaje; Envejecer; Hamlet, príncipe de Dinamarca; El mercader de Venecia; Los muñecos; Nena Teruel; Nicolás; El obscuro dominio; La romanza del vivir; El sexo débil; Sin querer; El zapatero y el rey.

8.6. De nuevo al frente de la Compañía Morano regresó este actor para ofrecer su trabajo al público logroñés. Realizó una serie de funciones en el teatro Moderno, entre el 2 y el 14 de febrero de 1915.

a) Elenco:

Actores: Aguado, Campos, Cobeña, Latorre, Francisco Morano, Emilio Portes, Rivelles, Rodríguez.

Actrices: Amparo Fernández Villegas, Juanita Gil Andrés, Pura Martín Gómez, María Santoncha.

b) Repertorio:

El alcalde de Zalamea; La calle de la Montera; El centenario; El chiquillo; La dama de las camelias; La garra; El intérprete de Hamlet; La llama- 
rada; Un milagro de San Antonio; Los muñecos; La otra vida; Papá Lebonnard; Raffles, el ladrón elegante; Rosas de otoño; Los semidioses; La Tizona; La vida es sueño; La zancadilla; El zapatero y el rey; Zaragüeta.

8.7. Regresó Morano al escenario del Bretón entre el 20 de septiembre y el 6 de octubre de 1919; esta vez el elenco se denominaba Compañía de Alta Comedia y Dramas Morano.

\section{a) Elenco:}

Actores: Ernesto Álvarez, Nicolás Díaz-Perchicot, Antonio Gentil, Gonzalo Llorens, Francisco López Silva, Francisco Morano, Marcial Morano, Justo Norro, Antonio Pérez Indarte, Fernando Porredón (hijo), Fernando Sala, Antonio Vida.

Actrices: Amparo Fernández Villegas, Pura Fernández Villegas, Eugenia Fraile, Carmen L. Lagar, Raquel Martínez, Julia Sala, María Santoncha, Josefina Satorres, Ángeles Somavilla, Carmen Tejada, María Zarco.

b) Repertorio:

El abuelo; El alcalde de Zalamea; Amor salvaje; El cardenal; La castellana; La cena de las burlas; Cobardías; El collar de estrellas; La comedia del honor; La emboscada; En mitad del corazón; La espuma del champán; Felipe Derblay; Los incasables; Mister Beverley; Los muñecos; Las murallas de Jericó; El nido ajeno; Papá Lebonnard; La propia estimación; Punta de viuda; El santo; Señora ama; Traidor, inconfeso y mártir; Viejas leyes.

8.8. Entre el 7 y el 12 de diciembre de 1921 volvió la compañía Francisco Morano al teatro Bretón de Logroño.

a) Elenco:

Actores: Calvero, Cañizares, Gonzalo Llorens, Francisco Morano, Marcial Morano, Navajas, Fernando SalaVigo.

Actrices: Amparo Fernández Villegas, Meléndez, Sofía Morano, Ángeles Morano, Julia Sala, Ángeles Somovilla, Eloísa Vigo.

b) Repertorio:

Amor a oscuras; Amor y ciencia; La casa de los milagros; La casona; El centenario; El collar de estrellas; El condenado; Los gansos del Capitolio; 
El intruso; El loco Dios; Punta de viuda; Los resignadores; Traidor, inconfeso y mártir.

8.9. Como Compañía Cómico-dramática Francisco Morano esta vez, volvió este grupo de actores a ofrecer sus funciones en el Bretón, entre el 12 y el 17 de enero de 1924.

a) Elenco:

Actores: Francisco Morano (1. ${ }^{\text {er }}$ actor).

Actrices: Amparo Fernández Villegas (1. a actriz).

b) Repertorio:

El agua milagrosa; El alcalde de Zalamea; La casa de la alegría; El centenario; El collar de estrellas; Las cosas de Gómez; Los monigotes; Papá Lebonnard; Sansón; Shylok, el judío; Tierra muerta.

8.10. De nuevo en el Bretón, se presentó la Compañía Morano para ofrecer su trabajo al público logroñés, entre el 24 de febrero y el 2 de marzo de 1926.

a) Elenco:

Actores: Francisco Cabezón, Fernando Calvera, Francisco Fernández, Francisco Morano, Marcial Morano, César Muro, José Ontiveros, Enrique Ponte.

Actrices: Amparo Fernández Villegas, Fifí Morano, Felisa Torres, Eloísa Vigo.

b) Repertorio:

El agua milagrosa; ;Béseme usted...!; Los cómicos de la legua; La condesa María; El infierno de aquí; Tortosa y Soler; Volver a vivir.

8.11. Entre los días 10 y 16 de mayo de 1927, puso una serie de obras en el escenario anterior la Gran Compañía Morano.

a) Elenco:

Actores: José Cañizares, Miguel de Llano, Federico Morano, Francisco Morano, Marcial Morano, Enrique Ponte, Joaquín Puyol. 
Actrices: Amparo Fernández Villegas, Fifí Morano, Teodora Moreno, Ángeles Somovilla, Eloísa Vigo.

b) Repertorio:

El avaro; La emboscada; La musa loca; ¡Padre...!; Primavera en otoño; Volver a vivir; ¿Y después?

8.12. El mismo escenario del Bretón fue el elegido por la Compañía cómico-dramática Francisco Morano para presentar su trabajo entre el 22 y el 26 de enero de 1930.

a) Elenco:

Actores: Francisco Morano, Marcial Morano, César Muro, Avelino Nieto. Actrices: Isabel Calvo, Pilar Calvo, Amparo Fernández Villegas.

b) Repertorio:

El alcalde de Zalamea; El avaro; El chiquitín de la casa; La fierecilla domada; Parodi y cía.; Topacio; La última novela.

8.13. La última visita de este actor a Logroño durante estos cincuenta años fue de nuevo al mismo coliseo y con la denominación anterior de la compañía; las representaciones tuvieron lugar entre el 24 y el 29 de marzo de 1931.

a) Elenco:

Actores: Rafael Calvo, Francisco Gutiérrez, Miguel de Llano, Federico Morano, Francisco Morano, Marcial Morano, Avelino Nieto, José Ruste.

Actrices: Pilar Calvo, Amparo Fernández Villegas, Rosa Luisa Gorostegui, Julia Santero.

b) Repertorio:

Béseme usted; La espada del hidalgo; Manos de plata; El oro del diablo; Papá Gutiérrez; El señor marqués, mi yerno. 


\section{COMPAÑÍ́A RAMBAL}

9.1. La primera aparición de este actor en los escenarios logroñeses del siglo XX se produjo con la Compañía dramática española de obras policiacas, norteamericanas y de gran espectáculo Angulo-Rambal, dirigida por Francisco Comes. Las funciones tuvieron lugar en el teatro Bretón entre el 15 y el 23 de febrero de 1919.

a) Elenco:

Actores: José Burgos, Emilio Castrillo, Francisco Comes (1. ${ }^{\mathrm{er}}$ actor), Francisco Fernández, Vicente Grancha, Joaquín Huerta, Manuel Juan, Francisco Martí, Julio Muñoz, José Ratin, Eduardo Rocamora, Horacio Sociás.

Actrices: Magdalena Alemany, María Bassó, Carmen Collado, María Hurtado, Amparo Martí, Amparo Martínez, Julia Medina (1. actriz), María Quesada, Isabel Ventura, Isabel Zaragoza.

b) Repertorio:

Los amos del mar o la escuadra invisible; El guante rojo; El hombre invisible; La mano que mata o el fantasma de palacio; El robo del millón de francos; El secreto del doctor Hopssom o el fin de la mano negra; Los secretos de la corte de Verona o un crimen de lesa majestad; El silbido fatal; La venganza del ajusticiado; Zigomar contra Nick-Carter.

9.2. Al frente de la Compañía Rambal volvió este artista a actuar en el mismo escenario logroñes, entre el 18 y el 27 de noviembre de 1919.

a) Elenco:

Actores: Crespi, Cuenca, González, Marín, Montesinos, Enrique Rambal, Del Río.

Actrices: Luque, Marín, Márquez, Murillo, Pérez, Rafaela Rodríguez (1. ${ }^{\text {a }}$ actriz), Sánchez.

b) Repertorio:

Un crimen de lesa majestad; El hombre que está en todas partes; Lord Lister, ladrón de frac; El misterio de un crimen; La muñeca trágica; La perla imperial o el club del silencio; Secreto de confesión o los mohicanos de 
París; Los sembradores de frío; Simbad el marino; La sonata de la muerte; La tragedia de los reyes.

9.3. Actuó en el Bretón, entre el 9 y el 11 de diciembre de 1924, la Compañía de comedias de obras de espectáculo Enrique Rambal.

a) Elenco:

Actores: Enrique Rambal (1. ${ }^{\text {er }}$ actor).

b) Repertorio:

El carnet del diablo; Los cuatro jinetes del Apocalipsis; La señorita del servicio doméstico.

9.4. Como Compañía Rambal presentó este actor su trabajo entre el 26 y el 29 de mayo de 1925 en el teatro Bretón.

a) Elenco:

Actores: Enrique Rambal.

b) Repertorio:

El corredor de la muerte; El mártir del Calvario; París-Lyon-Mediterráneo; El silbido fatal.

9.5. Al citado escenario volvió este elenco bajo la denominación de Compañía de comedias, dramas y obras de gran espectáculo Rambal, entre el 26 de febrero y el 2 de marzo de 1927.

a) Elenco:

Actores: Enrique Rambal.

b) Repertorio:

Los caballeros de la niebla o el misterio de la alcoba nupcial; Genoveva de Brabante; El hombre que vendió su alma al diablo; El incendio de Roma; La leyenda del castillo. 
9.6. Con el mismo nombre de la compañía ofrecen estos artistas una serie de representaciones en el Bretón, entre el 9 y el 16 de enero de 1930.

a) Elenco:

Actores: Enrique Rambal, Constante Viñas.

Actrices: Cormena, Carolina Fernangómez, Pla, Puchol,Viñas.

b) Repertorio:

El conde de Montecristo; Enrique de Lagardère o el jorobado; Miguel Strogoff o el correo secreto del zar; Las mil y una noches; Los miserables; París-Lyon-Mediterráneo; 20.000 leguas de viaje submarino; ;Volga! ¡Volga!

9.7. De nuevo, al mismo teatro, regresa este elenco con el nombre de Compañía de grandes espectáculos Rambal, entre el 10 y el 13 de junio de 1935.

a) Elenco:

Actores: Enrique Rambal (1. ${ }^{\mathrm{er}}$ actor).

Actrices: Manolita Ruiz (1. actriz).

b) Repertorio:

Las dos huérfanas de París o el registro de la policía; Genoveva de Brabante; El jorobado o el caballero Enrique de Lagardère; La vuelta al mundo en 80 días.

9.8. Con la misma denominación de la compañía actuó este grupo teatral entre el 13 y el 16 de diciembre de 1935, de nuevo en el Bretón y bajo la dirección de Enrique López Alarcón.

a) Elenco:

Actores: Enrique Rambal.

Actrices: Rosa Luisa Gorostegui.

b) Repertorio:

Casanova, el galante aventurero; Los envenenadores o el corredor de la muerte; El médico de las locas; Miss Thery. 
9.9. La misma compañía volvió de nuevo a Logroño, esta vez al cine Olimpia, entre el 12 y el 14 de febrero de 1936.

a) Elenco:

Actores: Enrique Rambal.

b) Repertorio:

Magdalena o la mujer adúltera; Secreto de confesión o los mohicanos de París; Sin novedad en el frente.

9.10. Volvió de nuevo Enrique Rambal al teatro Bretón dando nombre a la misma compañía, entre el 21 y el 25 de septiembre de 1939.

a) Elenco:

Actores: Enrique Rambal.

b) Repertorio:

Las dos huérfanas de París; Genoveva de Brabante; El jorobado o el caballero Enrique de Lagardère; Miguel Strogoff o el correo del zar; Los mohicanos de París o secreto mortal.

9.11. Con la misma denominación, esta compañía ofreció otra serie de representaciones en el mismo escenario, entre el 14 y el 22 de mayo de 1941.

a) Elenco:

Actores: Cabré, Enrique Rambal, Rupert, Enrique Vilches.

Actrices: Carmen Sánchez (1. actriz).

b) Repertorio:

Agustina de Aragón; Claustro y mundo; Fabiola o los mártires cristianos; Genoveva de Brabante; Isabel la Católica; Jonathan o el monstruo invisible; El jorobado o el caballero Enrique de Lagardère; Marco Antonio y Cleopatra; Las mil y una noches.

9.12. Entre el 2 y el 6 de octubre de 1944, regresó esta compañía al teatro Bretón para ofrecer al público logroñés varias funciones. 
a) Elenco:

Actores: Enrique Rambal.

b) Repertorio:

Don César el aventurero; Un guante rojo; París-Lyon-Mediterráneo; El prisionero de Zenda; Rebeca.

9.13. Los logroñeses pudieron ver de nuevo, en el mismo coliseo, a esta compañía entre el 20 y el 28 de octubre de 1945.

a) Elenco:

Actores: Burgos, Davi, Enrique Rambal, Ramos.

Actrices: Enriqueta Rambal.

b) Repertorio:

Calasanz; El conde de Montecristo; Las cuatro plumas; Drácula; Genoveva de Brabante; El incendio de Roma; El jorobado o el caballero Enrique de Lagardère; Kismet (el destino); Miguel Strogoff o el correo del zar.

9.14. La última visita de esta compañía a nuestra ciudad, durante estos cincuenta años, se produjo entre el 2 y el 13 de julio de 1947, nuevamente en el teatro Bretón.

a) Elenco:

Actores: José Cañizares, Fernando Carrasco, Francisco Fernández, Miguel Ibáñez, Enrique Rambal (padre e hijo), Ramos, José M. ${ }^{a}$ del Val.

Actrices: Mercedes Borque, Pla, Enriqueta Rambal, Amparo Reyes, M. ${ }^{\mathrm{a}}$ Carmen Unceta.

b) Repertorio:

Las cuatro plumas; Chu-chin-chow; Las dos huérfanas de París; Fabiola o los mártires cristianos; Genoveva de Brabante; Guerreras rojas; El jorobado o el caballero Enrique de Lagardère; Los mercaderes de ébano o la cabaña del negro Tom; Miguel Strogoff o el correo secreto del zar; El prisionero de Zenda; Rebeca. 


\section{COMPAÑÍA XIRGU}

10.1. Al frente de la Compañía Dramática Española Margarita Xirgu, apareció esta actriz por primera vez en Logroño en el año 1914, entre los días 25 y 30 de abril, para presentar sus puestas en escena en el teatro Bretón.

a) Elenco:

Actores: Francisco Barraycoa, Manuel Fernández, Eduardo Górriz, José Lucio, Vicente Moralca, Julio Ordóñez, Miguel Ortiz, Ricardo Puga, José Rivero, Leovigildo Ruiz Tatay, Fernando Sala, Juan Soler, Fernando Villalonga.

Actrices: Amparo Álvarez Segura, Adela Coy, Cecilia Coy, Celia Ortiz, María de las Rivas, Julia Sala, Josefina Santolaria, Josefa Segura, Margarita Xirgu.

b) Repertorio:

L' Aigrette (emblema de honor); Camino adelante; Electra; Los ojos de los muertos; El patio azul; Los románticos; La sangre gorda; El secreto.

10.2. Con la misma denominación volvió esta compañía al mismo coliseo logroñés, entre el 24 y el 30 de noviembre de 1914.

a) Elenco:

Actores: Francisco Barraycoa, Pedro Cabré, Manuel Fernández, Federico Górriz, José Lucio, Vicente Morales, Julio Ordóñez, Miguel Ortiz, Ramón Puga, Ricardo Puga, José Rivero, Fernando Sala, José Soler.

Actrices: Amparo Álvarez Segura, Concepción Ester, Amparo López, Guillermina Moreno, Celia Ortiz, Julia Riaza, María de las Rivas, Julia Sala, Josefina Santaolaria, Josefa Segura, Margarita Xirgu.

b) Repertorio:

Alma triunfante; La comida de las fieras; El corazón manda; Madame Pepita; Magda; La malquerida; Primerose; Zaza.

10.3. De nuevo, en el Bretón, ofreció una serie de obras esta actriz al frente esta vez de la Compañía Margarita Xirgu; sucedió entre el 21 y el 30 de septiembre de 1915. 
a) Elenco:

Actores: Francisco Barraycoa, Pedro Cabré, Enrique Casanova, Federico Górriz, José Lucio, Vicente Morales, Miguel Ortín, Ramón Puga, Ricardo Puga, José Rivero, Carlos Segura, José Soler.

Actrices: Amparo Álvarez, Concepción Ester, Amparo López, Guillermina Moreno, Celia Ortiz, Julia Riaza, María de las Rivas, Adela Santolaria, Josefina Santolaria, Josefa Segura, Dolores Valero, Margarita Xirgu.

b) Repertorio:

El amor tardio; El corazón manda; La cortina verde; La dama de las camelias; Frou-frou; La gata de angora; La marcha nupcial; La mujer desnuda; Primerose; La redacción; La reja; Sangre gorda; El tercer marido; Zaza.

10.4. Visitó el mismo escenario esta compañía de nuevo entre el 21 y el 30 de septiembre de 1920.

a) Elenco:

Actores: José Lucio, Alfonso Muñoz, Rivas, José Rivero.

Actrices: Amparo Álvarez Segura, Brú, Margarita Xirgu.

b) Repertorio:

Alimaña; La calumniada; La casa en orden; La chiquilla; La dama de las camelias; Fedora; La loca de la casa; Madame Pepita; El mal que nos hacen; Marianela; María Rosa; Primerose.

10.5. Entre el 19 de septiembre y el 1 de octubre de 1925 se presentó de nuevo la Compañía Dramática Española Margarita Xirgu, en el escenario del Bretón logroñés.

a) Elenco:

Actores: López Silva, Alfonso Muñoz (1. ${ }^{\mathrm{er}}$ actor).

Actrices: Carmen Carbonell, Margarita Xirgu.

b) Repertorio:

Campo de armiño; Cancionera; Carmen; Como antes, mejor que antes; El corazón manda; Cristalina; La dama de las camelias; Magda; Mariana; 
La mujer desnuda; La noche del sábado; La pecadora; Primerose; La prueba de la felicidad; Rosas de otoño; Thien-Hoa (Flor del cielo); La virtud sospechosa; Zaza.

10.6. Otra vez en el Bretón, el público logroñés puedo contemplar el trabajo de esta actriz, ahora en la Gran Compañía Dramática Xirgu-Borrás; las funciones fueron entre el 2 y el 3 de octubre de 1934.

a) Elenco:

Actores: Enrique Borrás, Alberto Contreras, Enrique A. Diosdado, Pedro López Lagar.

Actrices: Pilar Muñoz, Margarita Xirgu.

b) Repertorio:

Ni al amor ni al mar; Tierra baja.

10.7. La última aparición durante este periodo en Logroño de esta actriz fue entre el 11 y el 15 de enero de 1936, al frente de la Compañía Dramática Margarita Xirgu, bajo la dirección de Cipriano Rivas Cherif; de nuevo desarrolló su trabajo en el teatro Bretón.

a) Elenco:

Actores: Enrique Á. Diosdado, Alberto Contreras, Pedro López Lagar, Alejandro Maximino.

Actrices: Antonia Calderón, Luisa Calderón, Isabel Pradas, Amalia Sánchez Ariño, Amelia de la Torre, Eloísa Vigo, Margarita Xirgu.

b) Repertorio:

Bodas de sangre; La dama boba; Doña Rosita la soltera o el lenguaje de las flores; La sirena varada; Yerma.

Para finalizar haremos algunas puntualizaciones sobre lo expuesto hasta ahora. Respecto a los elencos, hay que observar que sólo en escasas ocasiones encontramos la publicación de las listas de las compañías en las fuentes manejadas, por ello hay que obtener los nombres de los actores de las escasas reseñas críticas que aparecen en las mismas. Evidentemente, la atención prestada no es la misma para todas las compañías, por eso unos elencos los 
tenemos completos, mientras que en otros apenas conocemos más que el nombre del titular de la compañía, como en el caso de Rambal, por ejemplo. Encontramos actores titulares de estas compañías que también han formado parte de otros elencos, como Francisco Fuentes en la compañía de Rosario Pino, en mayo de 1915, o María Bassó y Amparo Martí en la compañía de Rambal en febrero de 1919.

Un hecho que no queremos olvidar es el de la compañía Guerrero-Mendoza; tomando sólo el nombre de la compañía, tendríamos que haberla incluido en nuestra reseña, ya que visitaron Logroño en siete ocasiones. Sin embargo, no la hemos relacionado porque en las dos últimas ocasiones (1935 y 1941), los actores María Guerrero y Fernando Díaz de Mendoza ya habían muerto y los titulares de la compañía eran su hijo Fernando Díaz de Mendoza Guerrero y su sobrina María Guerrero López, que también formaban un matrimonio. Esto ocurre también con las compañías de Francisco Morano y de José Montijano, en las que sus hijos continúan la labor artística de sus progenitores.

En cuanto a los repertorios, ofrecemos sólo las obras que fueron representadas en nuestra ciudad y no la lista de obras que podía hacer cada compañía. Un hecho curioso en este sentido es la puesta en escena de dos obras de carácter lírico (Los campesinos y Ruido de campanas) por la compañía de Montijano, el 28 de diciembre de 1918, a modo de «inocentada», pues no era éste el género cultivado por estos actores ${ }^{1}$.

${ }^{1}$ Este trabajo es producto de mi tesis de doctorado, El teatro en Logroño (1850-1900) —que puede consultarse en «Estudios sobre teatro», en http://www.uned.es/centro-investigación-SELITEN@T—; así como se une a los nueve trabajos recopilados por José Romera Castillo (ed.), «En torno a la semiótica teatral: sobre algunas compañías profesionales en diversas ciudades españolas», Signa 12 (2003), 323-546 — también en http://cervantesvirtual.com/hemeroteca/signa/— y al de Inmaculada Benito Argáiz, «Compañías teatrales profesionales en Logroño (1850-1900)», Signa 14 (2005), 181-211. Cf. además lo señalado sobre la presencia de los dramaturgos áureos en los escenarios de Logroño en los dos artículos que se publican en el «Estado de la cuestión» que aparece al principio de este número de Signa. 